Fig. 1: Network visualization of the interaction of gene co-expression in the module best correlated with achieving sustained drug-free remission. Upregulated genes are expressed as green nodes and the downregulate gene as red node. The three rounded rectangular nodes display the highest co-expressed genes within the module ( $>10$ connections) when applying a weight cut-off of 0.01 . The average number of nodes connected at this cut-off was 4.6 (correlation coefficient 0.82 ).

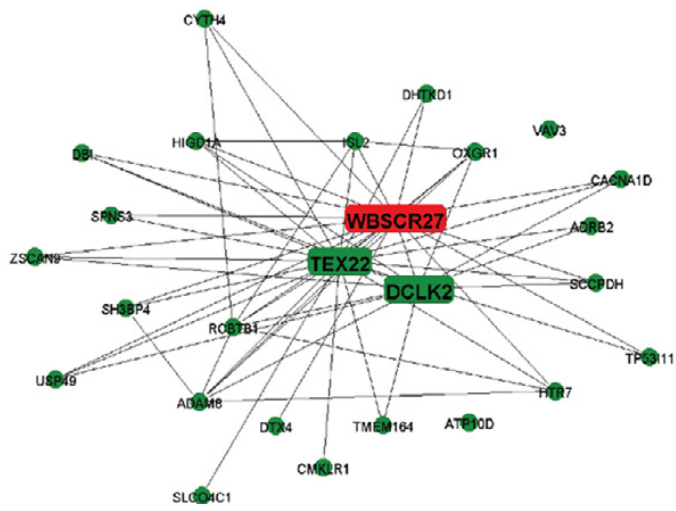

In addition, we identified 83 overrepresented Gene Ontology (GO) terms of which granulocyte migration $\left(p=2.70 \mathrm{E}^{-04}\right)$, myeloid leukocyte migration $\left(p=8.95 \mathrm{E}^{-04}\right)$ and G-protein coupled amine receptor activity $\left(p=1.25 \mathrm{E}^{-03}\right)$ were most significant. The genes in the module of interest showing the highest connectivity were the upregulated testis expressed 22 (TEX22), doublecortin lie kinase 2 (DCLK2), and the downregulated Williams Beuren syndrome chromosome region 27 (WBSCR27) gene (Fig. 1).

Conclusions: When performing network analyses of the DEGs between responders and non-responders, TEX22 and DCLK2 were identified as signature genes for treatment response to TCZ therapy. WBSCR27 was found to be associated with less chance of achieving sDFR.

References:

[1] Bijlsma JW, Welsing PM, Woodworth TG, et al. Early rheumatoid arthritis treated with tocilizumab, methotrexate, or their combination (U-Act-Early): a multicentre, randomised, double-blind, double-dummy, strategy trial. Lancet 2016; 388:343-55.

Disclosure of Interest: X. Teitsma: None declared, J. Jacobs: None declared, M. Mokry: None declared, A. Pethö-Schramm Employee of: F. Hoffmann-La Roche, M. Borm Employee of: Roche Nederland B.V., J. van Laar Consultant for: Received fees from MSD, Pfizer, Roche, Eli Lilly and BMS, J. Bijlsma Grant/research support from: Received research grants (to his department) and consultancy fees from AbbVie, BMS, Crescendo, MSD, Mundipharma, Pfizer, Roche, Sun and UCB, F. Lafeber: None declared

DOI: 10.1136/annrheumdis-2017-eular.2197

\section{OP0294 FRACTURE PREDICTION USING A GENETIC MARKERS ALGORITHM COMPARED TO FRAX IN THREE EUROPEAN COHORTS}

S. Ferrari ${ }^{1}$, R. Rizzoli ${ }^{1}$, R. Chapurlat ${ }^{2}$, M.L. Brandi ${ }^{3}$, H. Martínez ${ }^{4}$, M. Herrero ${ }^{4}$, J. Vergés $^{5}$, M. Artieda ${ }^{6}$, D. Tejedor ${ }^{6}$, A. Martínez $^{6}$, J. Blanch ${ }^{7}$, S. Palacios ${ }^{8}$. ${ }^{1}$ Geneva University Hospital and Faculty of Medicine, Geneve, Switzerland; ${ }^{2}$ Division of Rheumatology, INSERM U1033, Université de Lyon, Hôpital e Herriot, Lyon, France; ${ }^{3}$ University of Florence, Florence, Italy; ${ }^{4}$ Clinical $R \& D$, Bioiberica; ${ }^{5}$ Osteoarthritis Foundation International (OAFI), Barcelona; ${ }^{6} R \& D$ Department, Progenika Biopharma, A Grifols Company, Derio; ${ }^{7}$ Hospital del Mar of Barcelona, Barcelona; ${ }^{8}$ Palacios Institute of Health and Woman Medicine, Madrid, Spain

Background: Numerous genome-wide association studies (GWAS) and large meta-analyses have started to unravel the multiple gene polymorphisms associated with BMD and/or fragility fractures. However the clinical utility of these genetic markers for fracture prediction remains to be established.

Objectives: To develop a DNA genotyping tool for predicting osteoporotic fractures in postmenopausal women.

Methods: 768 SNPs previously associated with osteoporosis phenotypes were identified in silico through the NHGRI GWAS catalog and BoneKey Genetics website. They were genotyped on an Illumina GoldenGate assay in 1649 postmenopausal women aged 45+ yrs belonging to three osteoporotic fractures cohorts from Switzerland, Italy and France. SNPs potentially associated $(p<0.10)$ with prevalent and incident clinical fragility fractures in one or more of the cohorts, or in the cohorts together, were then combined in a genetic risk score (GRS). GRS association with fragility fractures was tested by forward logistic regressions adjusting for age and FN BMD. The ability of GRS for fracture prediction was evaluated by the area under the ROC curve (AUC) in the three cohorts combined, as well separately (for internal replication). For comparison, fracture probabilities were computed using FRAX clinical risk factors (without BMD) without and with the addition of GRS.

Results: The average prevalence of fragility fractures in the three cohorts was $25 \%$ (range 22 to $28 \%$ ), of which half were major fractures (FRAX definition). After
QC filtering, 632 SNPs in 1625 individuals were correctly genotyped, of which 73 were potentially associated with fractures in one or more cohorts. In single and multiple regression models, GRS was significantly associated with fractures (OR 1.09, Cl 1.07-1.12, $\mathrm{p}<0.0001$ ). The GRS AUC for fracture prediction was significant (0.65) and highly consistent among the three cohorts. GRS predicted major fractures as well as FRAX clinical risk factors without BMD (AUC 0.63 vs $0.58, p=0.08$ ), and when combined with clinical FRAX, the AUC was significantly improved $(0.67, \mathrm{p}=0.0106)$.

Conclusions: SNPs previously associated with osteoporosis phenotypes through large GWAS and meta-analyses can be replicated for association with fragility fractures in post-menopausal women from three European countries. Our results provide a proof-of-principle that a genetic risk score (GRS) based on these SNPS represents an independent risk factor for fractures and could be developed into a genetic algorithm to improve the prediction of fragility fractures, either alone or together with FRAX.

Disclosure of Interest: None declared

DOI: 10.1136/annrheumdis-2017-eular.6316

\section{OP0295 UNIQUE WHOLE BLOOD MICRORNA BIOSIGNATURE FOR RHEUMATOID ARTHRITIS}

V. Anaparti ${ }^{1,2,3}$, I. Smolik ${ }^{1,3}$, X. Meng ${ }^{1,2,3}$, N. Mookherjee ${ }^{2,3}$,

H. El-Gabalawy ${ }^{1,2,3} .{ }^{1}$ Rheumatic Diseases Unit; ${ }^{2}$ Manitoba Center for

Proteomics and Systems Biology; ${ }^{3}$ Internal Medicine, University of Manitoba,

Winnipeg, Canada

Background: RA susceptibility risk is disproportionately high ( $2-3$ fold) in Indigenous North American (INA) tribes compared to other populations ${ }^{1,2}$. Environmental, lifestyle \& genetic factors account for $<20 \%$ of observed disease variance suggesting contribution of additional risk determinants ${ }^{3}$. Emerging evidence suggests small non-coding microRNAs (miRs) e.g. miR-155, miR-146a, miR-26b are key contributors to RA pathogenesis ${ }^{4}$. In this project, we examined the role of miRs on RA incidence, and association with anti-citrullinated protein antibodies (ACPA), whose appearance precedes disease symptoms. We hypothesized that differential expression of specific miRs associated with disease symptoms will facilitate RA transition in genetically susceptible first-degree relatives (FDRs).

Methods: Whole blood and peripheral blood mononuclear cells (PBMCs) were obtained from age-matched ACPA+RA patients ( $n=18$ ), non-symptomatic ACPA+ FDRs $(n=12)$ and ACPA- healthy controls $(n=12)$, who belonged exclusively to INA Cree-Ojibway communities of Northern Manitoba, Canada. Total RNA was isolated using miRVANA kit (Ambion). Expression of selected $32 \mathrm{miRs}$ based on the published literature, and associated downstream mRNA targets, were monitored by quantitative real-time PCR, RNU48 and 18sRNA were used for input normalization for miRNA and mRNA expression respectively.

Results: Whole blood expression profiling identified 10 differentially expressed miRs in RA patients compared to control subjects. Expression of miR-103a-3p was significantly up-regulated ( $\sim 2.3$-fold; $p=0.0062)$, whereas that of imiR-16, miR-24, miR-29a, miR-125a-3p, miR-203, miR-222, miR-223, miR-150 and miR-346 were down-regulated in RA patients compared to controls. Increased miR-103a-3p expression was also confirmed in PBMCs from ACPA+ RA patients ( 2 fold; $\mathrm{p}=0.0040$ ) compared to controls. Further, miR-103a-3p expression was increased in ACPA+ FDRs ( $>4$ fold) compared to controls $(p=0.0005)$, and ACPA+ RA patients $(p=0.0149)$. miR-103a-3p expression was consistently elevated in ACPA+ FDRs, when we analyzed samples obtained at two independent time points (1year apart). Consistent with this, expression of AGO1 and DAPK1 mRNA, downstream targets of miR-103a-3p, was decreased significantly $(p<0.05)$ in ACPA+ FDRs compared to controls.

Conclusions: This study defines a unique signature of dysregulated miRs amongst RA patients and their related FDRs within the INA cohort. Our results suggest a potential role of miR-103a-3p as a prognostic biomarker for pre-clinical RA.

References:

[1] Barnabe et al J Rheumatol. 2008;35(6):1145-50.

[2] Smolik et al J Rheumatol. 2013;40(6):818-24.

[3] Viatte et al Nat Rev Rheumatol. 2013;9(3):141-53.

[4] Vicente et al Nat Rev Rheumatol. 2016 Apr;12(4):211-20.

Acknowledgements: Canadian Institute of Health \& Research (CIHR).

Research Manitoba

Disclosure of Interest: None declared

DOI: 10.1136/annrheumdis-2017-eular.1480

\section{OP0296 AUTOIMMUNE ASSOCIATED GENE PTPN22 NEGATIVELY REGULATES DECTIN-1 SIGNALLING IN DENDRITIC CELLS}

H. Purvis $^{1}$, F. Clarke ${ }^{1}$, C. Jordan ${ }^{1}$, C. Sanchez-Blanco ${ }^{1}$, G.H. Cornish ${ }^{1}$, D. Rawlings ${ }^{2}$, R. Zamoyska ${ }^{3}$, A.P. Cope ${ }^{1} .{ }^{1}$ Academic Department of Rheumatology, King's College London, London, United Kingdom; ${ }^{2}$ Seattle Children's Research Institute and Departments of Pediatrics and Immunology, University of Washington School of Medicine, Seattle, United States; ${ }^{3}$ Institute of Immunology and Infection Research, Edinburgh University, Edinburgh, United Kingdom

Background: A single nucleotide polymorphism within the phosphatase PTPN22 
increases the risk of developing multiple autoimmune and connective tissue diseases. ${ }^{1}$ Ptpn22 is a negative regulator of Syk and Src family kinases downstream of immuno-receptor signalling cascades. ${ }^{2}$ Fungal $\beta$-glucan receptor dectin-1, signals via Syk kinase, and induces dendritic cells to secrete proinflammatory cytokines IL-1 $\beta, \mathrm{IL}-6, \mathrm{IL}-12 / 23 p 40$ and $\mathrm{TNF} \alpha$, in turn allowing the induction of IL-17 secreting T-cell responses, which are critical to the clearance of fungal infections. ${ }^{3} \mathrm{IL}-17$ has been implicated as a key cytokine in inflammatory responses associated with RA, JIA, and psoriasis. ${ }^{4}$

Objectives: To investigate if Ptpn22 regulates dectin-1 signalling and controls the capability of dectin-1 matured BMDC to promote adaptive immune responses. Methods: GM-CSF bone marrow derived dendritic cells (BMDC) were generated from C57BL/6 WT, Ptpn22-- or Ptpn22 ${ }^{\mathrm{R} 619 \mathrm{~W}}$ (human PTPN22 ${ }^{\mathrm{R} 620 \mathrm{~W}}$ orthologue) mice, and pulsed with $\mathrm{OVA}_{323-339}$ in the presence or absence of the dectin-1 agonist curdlan. Activated BMDC were co-cultured in vitro with OT-II T-cells or adoptively transferred into OT-II mice and the resulting T-cell response assessed. Cytokine secretion from curdlan activated Ptpn22 variant mouse BMDC was determined by immunoassay and the kinetics of Syk and Erk phosphorylation were determined by immunoblot.

Results: We observed that Dectin-1 activated Ptpn22-- BMDC had an enhanced capability to induce T-cell IL-17 secretion both in vitro and in vivo compared to WT BMDC. Following dectin-1 priming Ptpn22 ${ }^{-/}$BMDC secreted increased IL-1 $\beta$ compared to WT BMDC, and the increase in IL-1 $\beta$ was found to be sufficient to cause the enhanced IL-17 response induced by Ptpn22 $2^{-1}$ BMDC. Dectin- 1 induced IL-1 $\beta$ secretion was found to be Syk and Erk dependent and assessment of Syk and Erk kinetics of phosphorylation revealed that dectin-1 activated Ptpn22-1BMDC displayed enhanced Syk and Erk phosphorylation compared to WT BMDC. Furthermore, Ptpn22 ${ }^{\mathrm{R} 619 \mathrm{~W}}$ BMDC (orthologue of human Ptpn22 ${ }^{\mathrm{R} 620 \mathrm{~W}}$ ) exhibited a similar enhancement in IL-1 $\beta$ secretion and induced enhanced T-cell dependent IL-17 responses in vivo, indicating that the PTPN22 polymorphism behaves as a loss-of-function allele in the context of dectin-1 signals.

Conclusions: Data highlight Ptpn22 as a novel regulator of dectin-1 signals and provide a link between genetically conferred perturbation to innate receptor signalling pathways and autoimmunity.

References:

[1] Burn, G. L., Svensson, L., Sanchez-Blanco, C., Saini, M. \& Cope, A. P. Why is PTPN22 a good candidate susceptibility gene for autoimmune disease? FEBS Lett. 585, 3689-98 (2011).

[2] Cloutier, J. F. \& Veillette, A. Cooperative inhibition of T-cell antigen receptor signaling by a complex between a kinase and a phosphatase. J. Exp. Med. 189, 111-21 (1999).

[3] Dambuza, I. M. \& Brown, G. D. C-type lectins in immunity: recent developments. Curr. Opin. Immunol. 32, 21-27 (2015).

[4] Tabarkiewicz, J., Pogoda, K., Karczmarczyk, A., Pozarowski, P. \& Giannopoulos, K. The Role of IL-17 and Th17 Lymphocytes in Autoimmune Diseases. Arch. Immunol. Ther. Exp. (Warsz). 63, 435-49 (2015).

Disclosure of Interest: None declared

DOI: 10.1136/annrheumdis-2017-eular.4717

\section{OP0297 FUNCTIONAL POLYMORPHISMS OF THE ABCG2 GENE IN PRIMARY GOUT}

B. Stiburkova ${ }^{1,2}$, K. Pavelcova ${ }^{3,4}$, L. Petru ${ }^{3,4}$, P. Cepek ${ }^{3}$, J. Zavada ${ }^{3}$, K. Pavelka ${ }^{3}{ }^{1}$ Institute of Rheumatology, Prague $2 ;{ }^{2}$ Institute of Inherited Metabolic Disorders, First Faculty of Medicine, Charles University; ${ }^{3}$ Institute of Rheumatology; ${ }^{4}$ Department of Rheumatology, First Faculty of Medicine, Charles University, Prague, Czech Republic

Background: The urate transporters are one of the genetic determinants of serum uric acid concentrations. Common dysfunctional variants of $A B C G 2$ are revealed to be a major cause of gout and hyperuricemia by decreasing urate excretion. In this study we describe the analysis of allelic variants in the ABCG2 in a cohort with primary gout.

Objectives: The cohort was recruited in the Institute of Rheumatology, Prague. Gouty arthritis was diagnosed in 146 subjects (132 men, 14 women) according to the American college of rheumatology criteria 1977. Patients suffering from secondary gout and other purine metabolic disorders associated with pathological concentrations of serum uric acid were excluded.

Methods: In each patient, the family history of gout, number of gouty attacks, the presence of tophi, the occurrence of associated diseases and therapy have been assessed. All 16 exons of ABCG2 were amplified using PCR, and sequenced directly.

Results: In the $A B C G 2$ gene, 19 intronic sequence variants were detected. In the case of $c \cdot 689+1 \mathrm{G}>\mathrm{A}$, related to an individual with severe gouty phenotype, two abnormal splicing variants were identified: a) r.[532_689del]; b) r.[532 689del], r.[944 949del]. Identified deletions lead to frameshift and premature stop codon introduction ${ }^{1}$. From the 12 exon variants detected, there were nine non-synonymous: p.V12M, p.Q141K, p.R147W, p.T153M, p.F373C, p.T421A, p.T434M, p.S476P and p.D620N. Heterozygous p.V12M was detected in seven individuals. Heterozygous variants p.R147W, p.T153M, p.F373C, p.T421A, p.T434M and p.S476P were detected once, variant p.D620N twice. All these allelic variants were in silico predicted as a probably damaging. The p.Q141K, previously functionally characterized allelic variant with a strong effect on uric acid secretion impairment, was in cohort of gout patients presented with significantly higher minor allele frequency 0.24 (MAF), 57 heterozygotes/6 homozygotes, than in population of European origin $(\mathrm{MAF}=0.09)$ and world-wide population (MAF=0.12). In our cohort, the age of onset of gout had a normal distribution. Remarkably, in $75 \%$ of all individuals with early onset of gout between age 10-20 years, we detected allelic variant p.Q141K (in 6 out of 8 patients). In the age group $21-30$ years, this allelic variant was detected in $42 \%$ of patients (8/19), and in the age group $61-70$ years, just in $29 \%$ (13/29).

Conclusions: Our results show that genetic factor $A B C G 2$ should be considered as one of the strong common risks for gout. In summary, we revealed that allelic variants of $A B C G 2$, especially dysfunctional variant p.Q141K, have a significant effect on earlier onset of gout.

\section{References:}

[1] Stiburkova B et al. Novel dysfunctional variant in ABCG2 as a cause of severe tophaceous gout: biochemical, molecular genetics and functional analysis. Rheumatology (Oxford). 2016 Jan;55(1):191-4.

Acknowledgements: This study was supported by the grants from the Czech Republic Ministry of Health AZV 15-26693A.

Disclosure of Interest: None declared

DOI: 10.1136/annrheumdis-2017-eular.4901

\section{OP0298 CHROMATIN INTERACTIONS REVEAL NOVEL GENE TARGETS FOR DRUG REPOSITIONING IN RHEUMATIC DISEASES}

P. Martin ${ }^{1}$, K. Duffus ${ }^{1}$, A. McGovern ${ }^{1}$, A. Yarwood ${ }^{1}$, A. Barton ${ }^{1,2}$ J. Worthington ${ }^{1,2}$, S. Eyre ${ }^{1}$, G. Orozco ${ }^{1} .{ }^{1}$ Arthritis Research UK Centre for Genetics and Genomics, University of Manchester; ${ }^{2}$ NIHR Manchester Musculoskeletal Biomedical Research Unit, Central Manchester Foundation Trust, Manchester Academic Health Science Centre, Manchester, United Kingdom

Background: The treatment of rheumatic diseases can be both expensive and ineffective with up to $1 / 3$ of patient's failing to respond to current treatments. There is therefore a need to identify new treatments and to target these to individual patients. Although genetic studies have been successful in identifying common variation associated with disease susceptibility, a large proportion of these lie outside protein-coding regions. Many show enhancer activity but it is often unclear which gene(s) they regulate and how they contribute to disease. Chromatin folding brings linearly distant areas of the genome, such as promoters and enhancers, into close proximity, driving gene expression. Capture $\mathrm{Hi}-\mathrm{C}(\mathrm{CHi}-\mathrm{C})$ interrogates these interactions in a high-throughput, high-resolution manner, linking implicated enhancers to causal genes.

Objectives: Utilising our existing $\mathrm{CHi}-\mathrm{C}$ data on 3 rheumatic diseases, RA, JIA and PsA, targeting all known genetic associations, we explored the potential to identify candidate causal genes that are targets for existing drugs, which could be repositioned for use in these diseases.

Methods: Chromatin interaction data for $\mathrm{T}-$ and $\mathrm{B}$-cells in the 3 diseases was analysed using CHiCAGO v2 using a score cut-off of $\geq 5$. Interactions between disease regions and promoters were identified using BEDTOOLS v2.21.0 and intersected with drug targets from DrugBank v4.5.0. Existing treatments for each disease were identified by the presence of the relevant name in the "indication" field.

Results: Overall 850 genes were identified as interacting with a disease associated region. Of these, 61 are existing drug targets (303 drugs) (Table 1) and 9 are existing therapies used in the treatment of disease, primarily RA.

\begin{tabular}{lcccccc}
\multicolumn{6}{l}{ Table 1. Summary of drug targets identified by CHi-C } \\
\hline Disease & $\begin{array}{c}\text { Number of } \\
\text { identified } \\
\text { by CHi-C }\end{array}$ & $\begin{array}{c}\text { Number of } \\
\text { genes which } \\
\text { are existing } \\
\text { drug targets }\end{array}$ & $\begin{array}{c}\text { Number of } \\
\text { drugs } \\
\text { identified }\end{array}$ & $\begin{array}{c}\text { Number of } \\
\text { drugs } \\
\text { withdrawn }\end{array}$ & $\begin{array}{c}\text { Number of } \\
\text { drugs currently } \\
\text { used }\end{array}$ & $\begin{array}{c}\text { Number of drugs } \\
\text { for potential } \\
\text { repositioning }\end{array}$ \\
\hline RA & 510 & 41 & 106 & 4 & 9 & 93 \\
JIA & 324 & 16 & 74 & 0 & 1 & 73 \\
PSA & 196 & 13 & 147 & 0 & 0 & 147 \\
All & 850 & 61 & 303 & 4 & 9 & 290 \\
\hline
\end{tabular}

Conclusions: Our study identifies genes which are implicated in disease, are the target of existing drugs and offer the potential for drug repositioning. Of the potential drugs identified for RA, 14 are used in the treatment of various cancers and 11 are used in the treatment of diabetes and multiple sclerosis. Interestingly, 17 potential drugs identified for PsA are used in the treatment of schizophrenia and 11 in the treatment of hypertension. This data shows a novel insight into how functional annotation of genetic associations in rheumatic diseases can provide gene targets for re-positioned therapies.

Acknowledgements: This work was funded by Wellcome Trust Research Career Development Fellowship (095684); Arthritis Research UK (grant numbers 20385, 20571); Wellcome Trust (097820/Z/11/B); European Union's FP7 Health Programme (FP7-HEALTH-F2-2012-305549, Euro-TEAM, FP7/2007-2013); Innovative Medicines Initiative (BeTheCure project 115142); Medical Research Council (MR/K015346/1); National Institute for Health Research Manchester Musculoskeletal Biomedical Research Unit; Biotechnology and Biological Sciences Research Council UK (BBS/E/B/000C0405).

Disclosure of Interest: None declared

DOI: 10.1136/annrheumdis-2017-eular.2685 